TRANSACTIONS OF THE

AMERICAN MATHEMATICAL SOCIETY

Volume 363, Number 9, September 2011, Pages 4757-4776

S 0002-9947(2011)05239-6

Article electronically published on April 11, 2011

\title{
LOCAL RIGIDITY OF INVERSIVE DISTANCE CIRCLE PACKING
}

\author{
REN GUO
}

\begin{abstract}
A Euclidean (or hyperbolic) circle packing on a triangulated closed surface with prescribed inversive distance is locally determined by its cone angles. We prove this by establishing a variational principle.
\end{abstract}

\section{INTRODUCTION}

1.1. Andreev-Thurston Theorem. In his work on constructing hyperbolic metrics on 3-manifolds, W. Thurston (23, Chapter 13) studied a Euclidean (or hyperbolic) circle packing on a triangulated closed surface with prescribed intersection angles. Thurston's work generalizes Andreev's result of circle packing on a sphere [1, 2]. The special case of tangent circle packing (every intersection angle is zero) on a sphere was obtained by Koebe [16.

Suppose $(\Sigma, T)$ is a triangulated closed surface so that $V, E, F$ are sets of all vertices, edges and triangles in $T$. We identify vertices of $T$ with indexes, edges of $T$ with pairs of indexes and triangles of $T$ with triples of indexes. This means $V=\{1,2, \ldots,|V|\}, E=\{i j \mid i, j \in V\}$ and $F=\{\triangle i j k \mid i, j, k \in V\}$. Fix a vector $\Theta \in \mathbb{R}^{|E|}$ indexed by the set of edges $E$ such that $\Theta_{i j} \in\left[0, \frac{\pi}{2}\right]$ for each $i j \in E$. This vector is called a weight on $(\Sigma, T)$.

A circle packing on a weighted triangulated closed surface $(\Sigma, T, \Theta)$ is a configuration $\left\{c_{i}, i \in V\right\}$ of circles such that the intersection angle of $c_{i}$ and $c_{j}$ is $\Theta_{i j}$, which is the prescribed weight on the edge $i j$. To obtain a Euclidean (or hyperbolic) circle packing for $(\Sigma, T, \Theta)$, we start with a radius vector $r=\left(r_{1}, r_{2}, \ldots, r_{|V|}\right) \in \mathbb{R}_{>0}^{|V|}$ which assigns each vertex $i \in V$ a positive number $r_{i}$. A radius vector $r$ produces a Euclidean (or hyperbolic) cone metric on the surface as follows.

In Figure 1, consider a topological triangle $\triangle i j k$. One can construct a Euclidean (or hyperbolic) triangle $\triangle i j p$ such that the edges $i p, j p$ have lengths $r_{i}, r_{j}$ respectively and the angle at $p$ is $\pi-\Theta_{i j}$. Let $l_{i j}$ be the length of the edge $i j$ in the Euclidean (or hyperbolic) triangle $\triangle i j p$ which is a function of $r_{i}, r_{j}$. In fact, in Euclidean geometry

$$
l_{i j}=\sqrt{r_{i}^{2}+r_{j}^{2}+2 r_{i} r_{j} \cos \Theta_{i j}}
$$

or in hyperbolic geometry

$$
l_{i j}=\cosh ^{-1}\left(\cosh r_{i} \cosh r_{j}+\cos \Theta_{i j} \sinh r_{i} \sinh r_{j}\right) .
$$

Similarly, we obtain $l_{j k}, l_{k i}$.

Received by the editors April 2, 2009 and, in revised form, September 17, 2009.

2000 Mathematics Subject Classification. Primary 52C26, 58E30.

Key words and phrases. Circle packing, rigidity, variational principle.

(C)2011 American Mathematical Society 4757

Reverts to public domain 28 years from publication 


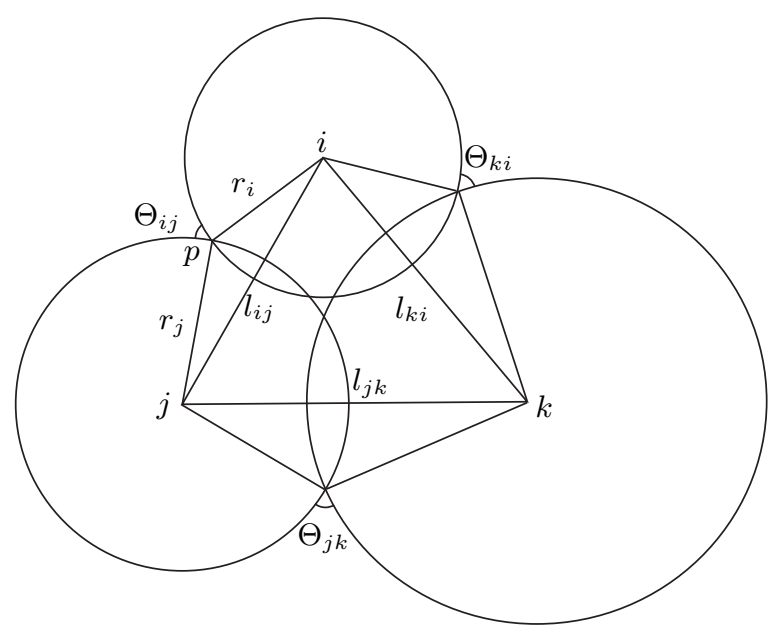

FIgURE 1. Andreev-Thurston circle packing

As observed by Thurston [23], under the assumption that $\Theta_{i j} \in\left[0, \frac{\pi}{2}\right]$ for each $i j \in E, l_{i j}, l_{j k}$ and $l_{k i}$ can be realized as edge lengths of a Euclidean (or hyperbolic) triangle $\triangle i j k$ in $F$. Gluing all of these Euclidean (or hyperbolic) triangles in $F$ produces a Euclidean (or hyperbolic) cone metric on $(\Sigma, T)$ with possible cones at vertices of $T$. On this surface with a cone metric, drawing a circle centered at vertex $i$ with radius $r_{i}$ for each vertex $i \in V$, we obtain a circle packing with prescribed intersection angles.

At each vertex $i \in V$, the cone angle $a_{i}$ is the sum of all inner angles having vertex $i$. Thus cone angles $\left(a_{1}, a_{2}, \ldots, a_{|V|}\right)$ are functions of the radius vector $r=\left(r_{1}, r_{2}, \ldots, r_{|V|}\right)$. We try to find a radius vector when cone angles are given. The Andreev-Thurston Theorem answers the question about the existence and uniqueness of radius vectors when cone angles are given.

Theorem 1 (Andreev-Thurston). For any weighted triangulated closed surface $(\Sigma, T, \Theta)$ with $\Theta_{i j} \in\left[0, \frac{\pi}{2}\right]$ for each $i j \in E$, a Euclidean (or hyperbolic) circle packing for $(\Sigma, T, \Theta)$ is determined by its cone angles up to Euclidean similarity (or hyperbolic isometry). Furthermore, the set of all possible cone angles form an open convex polytope in $\mathbb{R}^{|V|}$.

For a proof, see Thurston [23, Marden-Rodin [19], Colin de Verdiére [8], He [14, Chow-Luo 9], and Stephenson [22].

1.2. Inversive distance. H. S. M. Coxeter [7] introduced the notion of inversive distance to describe the relationship between two circles in a Möbius plane. The notion of inversive distance generalizes the notion of an intersection angle of two circles.

The inversive distance between two circles is independent of geometry, i.e., the inversive distance of two circles is the same under spherical, Euclidean and hyperbolic metrics. This generalizes the fact that the intersection angle of two circles is the same under spherical, Euclidean and hyperbolic metrics.

In the Euclidean plane, consider two circles $c_{1}, c_{2}$ with radii $r_{1}, r_{2}$ respectively. In the relevant case of this paper, we assume that $c_{i}$ does not contain $c_{j}$ for $\{i, j\}=$ 
$\{1,2\}$. If the distance between their center is $l$, the inversive distance between $c_{1}, c_{2}$ is given by the formula

$$
I\left(c_{1}, c_{2}\right)=\frac{l^{2}-r_{1}^{2}-r_{2}^{2}}{2 r_{1} r_{2}} .
$$

In hyperbolic plane, consider two circles $c_{1}, c_{2}$ with radii $r_{1}, r_{2}$ respectively. We assume that $c_{i}$ does not contain $c_{j}$ for $\{i, j\}=\{1,2\}$. If the distance between their center is $l$, the inversive distance between $c_{1}, c_{2}$ is given by the formula

$$
I\left(c_{1}, c_{2}\right)=\frac{\cosh l-\cosh r_{1} \cosh r_{2}}{\sinh r_{1} \sinh r_{2}} .
$$

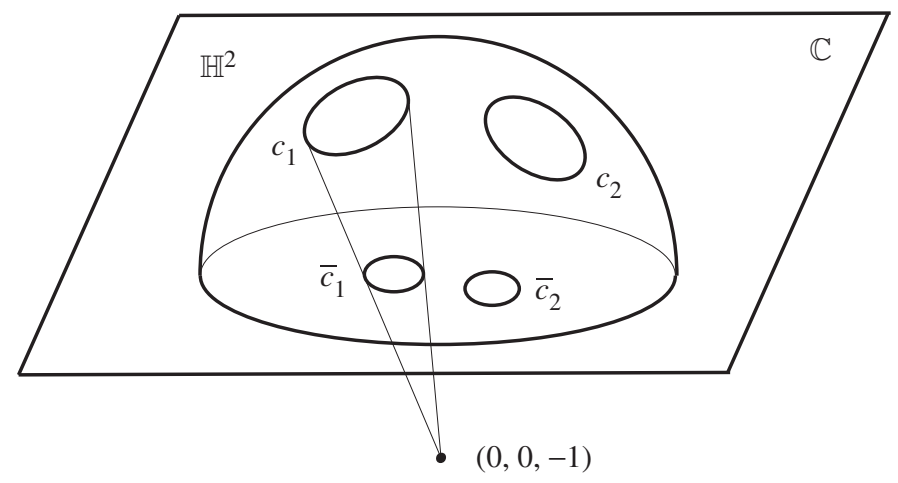

FiguRE 2. The stereographic projection

These two formulas are related by a stereographic projection. In Figure 2, consider the upper half space model of the hyperbolic space $\mathbb{H}^{3}$. The hyperbolic plane $\mathbb{H}^{2}$ is realized as a totally geodesic plane in $\mathbb{H}^{3}$, where it is a unit hemisphere perpendicular to the infinite boundary $\mathbb{C}$. The stereographic projection is the projection starting from point $(0,0,-1)$, which sends objects in $\mathbb{H}^{2}$ into $\mathbb{C}$. Let $c_{1}, c_{2}$ be two circles in $\mathbb{H}^{2}$ with radii $r_{1}, r_{2}$ and the distance between centers $l$. The stereographic projection produces two circles $\bar{c}_{1}, \bar{c}_{2}$ on the Euclidean plane $\mathbb{C}$ with radii $\bar{r}_{1}, \bar{r}_{2}$ and the distance between centers $\bar{l}$. Then we have

$$
I\left(c_{1}, c_{2}\right)=\frac{\cosh l-\cosh r_{1} \cosh r_{2}}{\sinh r_{1} \sinh r_{2}}=\frac{\bar{l}^{2}-\bar{r}_{1}^{2}-\bar{r}_{2}^{2}}{2 \bar{r}_{1} \bar{r}_{2}}=I\left(\bar{c}_{1}, \bar{c}_{2}\right) .
$$

We say that the stereographic projection preserves the inversive distance.

When $I\left(c_{1}, c_{2}\right) \in(-1,0)$, the circles $c_{1}, c_{2}$ intersect with an intersection angle $\arccos I\left(c_{1}, c_{2}\right) \in\left(\frac{\pi}{2}, \pi\right)$. When $I\left(c_{1}, c_{2}\right) \in[0,1)$, the circles $c_{1}, c_{2}$ intersect with an intersection angle $\arccos I\left(c_{1}, c_{2}\right) \in\left(0, \frac{\pi}{2}\right]$. When $I\left(c_{1}, c_{2}\right)=1$, the circles $c_{1}, c_{2}$ are tangent to each other. When $I\left(c_{1}, c_{2}\right) \in(1, \infty)$, the circles $c_{1}, c_{2}$ are separated.

1.3. Inversive distance circle packing. Motivated by the application to discrete conformal mappings, Bowers-Stephenson [5] introduced inversive distance circle packing which generalizes Andreev-Thurston's intersection angle circle packing. See Stephenson 22] (page 331) and Bowers-Hurdal [4] for more information. One of the 
important applications of inversive distance circle packing is the conformal mapping of the human brain; see, for example, Hurdal-Stephenson [15].

We will use the same notation as in Andreev-Thurston's intersection angle circle packing. For a triangulated closed surface $(\Sigma, T)$, fix a weight $I \in \mathbb{R}^{|E|}$ with $I_{i j} \in[0, \infty)$ for each $i j \in E$. In this paper, we will not consider the case of obtuse intersection angles. (Rivin 20] and Leibon [17 obtained results for certain circle patterns on surfaces allowing obtuse intersection angles.) An inversive distance circle packing for $(\Sigma, T, I)$ is a configuration of circles $\left\{c_{i}, i \in V\right\}$ such that the inversive distance of two circles $c_{i}, c_{j}$ is the prescribed number $I_{i j}$ for the edge $i j$. To obtain a Euclidean (or hyperbolic) circle packing for $(\Sigma, T, I)$, we start with a radius vector $r=\left(r_{1}, r_{2}, \ldots, r_{|V|}\right) \in \mathbb{R}_{>0}^{|V|}$ which assigns each vertex $i \in V$ a positive number $r_{i}$. A radius vector $r$ produces a Euclidean (or hyperbolic) cone metric on the surface as follows.

Consider a topological triangle $\triangle i j k \in F$. Assign a number $l_{i j}$ to the edge $i j \in E$, where $l_{i j}$ is the distance of two circles with radii $r_{i}, r_{j}$ and inversive distance $I_{i j}$. Therefore $l_{i j}$ is a function of $r_{i}, r_{j}$. In fact, in Euclidean geometry

$$
l_{i j}=\sqrt{r_{i}^{2}+r_{j}^{2}+2 r_{i} r_{j} I_{i j}}
$$

or in hyperbolic geometry

$$
l_{i j}=\cosh ^{-1}\left(\cosh r_{i} \cosh r_{j}+I_{i j} \sinh r_{i} \sinh r_{j}\right) .
$$

Similarly, we obtain $l_{j k}, l_{k i}$.

To guarantee that $l_{i j}, l_{j k}, l_{k i}$ satisfy the triangle inequality for each triangle $\triangle i j k \in F$, a vector $r=\left(r_{1}, r_{2}, \ldots, r_{|V|}\right)$ must satisfy certain conditions. For details, see sections 2.1 and 3.1.

For a radius vector $r$, if $l_{i j}, l_{j k}, l_{k i}$ can be realized as edge lengths of a Euclidean (or hyperbolic) triangle for each $\triangle i j k \in F$, gluing all of these Euclidean (or hyperbolic) triangles in $F$ produces a Euclidean (or hyperbolic) cone metric on $(\Sigma, T)$ with possible cones at vertices of $T$. On this surface with a cone metric, drawing a circle centered at vertex $i$ with radius $r_{i}$ for each vertex $i \in V$, we obtain a circle packing with prescribed inversive distance.

The cone angles $\left(a_{1}, a_{2}, \ldots, a_{|V|}\right)$ are functions of the radius vector $r=\left(r_{1}, r_{2}, \ldots\right.$, $\left.r_{|V|}\right)$. We are interested in the solutions of a radius vector when cone angles are given.

Our main theorem is the local rigidity of inversive distance circle packing.

Theorem 2. For any weighted triangulated closed surface $(\Sigma, T, I)$ with $I_{i j} \in[0, \infty)$ for each $i j \in E$, a Euclidean (or hyperbolic) inversive distance circle packing for $(\Sigma, T, I)$ is locally determined by its cone angles up to Euclidean similarity (or hyperbolic isometry).

The local rigidity of the Andreev-Thurston Theorem is generalized to the case of inversive distance circle packing. In our case, we are not able to obtain either the global rigidity or the description of the space of cone angles as in the AndreevThurston Theorem. For discussion, see sections 2.1 and 3.1.

In section 2, we prove Theorem 2 in Euclidean geometry. In section 3, we prove Theorem 2 in hyperbolic geometry. 
1.4. Variational principle. Theorem 2 is proved by establishing a variational principle. The energy function of this variational principle is derived from the derivative of the cosine law of a Euclidean (or hyperbolic) triangle. Andreev and Thurston obtained their results without using variational principles. The variational approach to circle packing was first introduced by Colin de Verdiére 8 . Since then, many works on variational principles on circle packing or circle patterns have appeared. For example, see Brägger [3], Rivin [20, Leibon [17, Chow-Luo 9], Bobenko-Springborn [6], Guo [12, Springborn [21], Luo [18, Guo-Luo [13] and others.

Applying a variational principle, Colin de Verdiére [8] proved the AndreevThurston Theorem in the special case of tangent circle packing, i.e., $\Theta_{i j}=0$ for each $i j \in E$. Chow-Luo [9] proved the Andreev-Thurston Theorem of intersection angle circle packing, i.e., $\Theta_{i j} \in\left[0, \frac{\pi}{2}\right]$ for each $i j \in E$. Variational principles for polyhedral surfaces including the topic of circle packing are studied systematically in Luo [18. Many energy functions are derived from the cosine law and its derivative. Tangent circle packing is generalized to tangent circle packing with a family of discrete curvature. For exposition of this work, see also Dai-Gu-Luo [10]. We follow this variational approach to prove Theorem 2 of inversive distance circle packing, i.e., $I_{i j} \in[0, \infty)$ for each $i j \in E$.

\section{Circle packing in Euclidean geometry}

2.1. Space of radius vectors. As stated in the Introduction, to obtain a Euclidean cone metric, we need conditions on a radius vector. In this section we investigate the space of radius vectors for one triangle in Euclidean geometry. Consider a topological triangle $\triangle i j k \in F$. Assign a number $l_{i j}$ to the edge $i j \in E$, where $l_{i j}$ is the distance of the centers of two circles with radii $r_{i}, r_{j}$ and inversive distance $I_{i j}$. Therefore $l_{i j}$ is a function of $r_{i}, r_{j}$. In fact, in Euclidean geometry

$$
l_{i j}=\sqrt{r_{i}^{2}+r_{j}^{2}+2 r_{i} r_{j} I_{i j}} .
$$

Similarly, we obtain $l_{j k}, l_{k i}$.

Lemma 3. The set $\mathcal{R}_{E}^{i j k}:=\left\{\left(r_{i}, r_{j}, r_{k}\right) \in \mathbb{R}_{>0}^{3} \mid l_{i j}, l_{j k}, l_{k i}\right.$ satisfy triangle inequality $\}$ is a connected and simply connected open subset in $\mathbb{R}_{>0}^{3}$.

Proof. Let $\rho_{E}: \mathbb{R}_{>0}^{3} \rightarrow \mathbb{R}_{>0}^{3}$ be the map sending $\left(r_{i}, r_{j}, r_{k}\right)$ to $\left(l_{i j}, l_{j k}, l_{k i}\right)$. We claim that $\rho_{E}$ is a smooth embedding. First the determinant of the Jacobian matrix of $\rho_{E}$ is nonzero. Next, we will show $\rho_{E}$ is one-to-one.

This means we need to show that if the following equations have a solution $\left(r_{i}, r_{j}, r_{k}\right)$, then the solution is unique:

$$
\begin{aligned}
r_{j}^{2}+r_{k}^{2}+2 I_{j k} r_{j} r_{k} & =l_{j k}^{2}, \\
r_{k}^{2}+r_{i}^{2}+2 I_{k i} r_{k} r_{i} & =l_{k i}^{2}, \\
r_{i}^{2}+r_{j}^{2}+2 I_{i j} r_{i} r_{j} & =l_{i j}^{2} .
\end{aligned}
$$

Solving the first equation for $r_{j}$, we get $r_{j}=-I_{j k} r_{k} \pm \sqrt{\left(I_{j k}^{2}-1\right) r_{k}^{2}+l_{j k}^{2}}$. Since $I_{j k}>0$ and $r_{j}>0$, we must have $r_{j}=-I_{j k} r_{k}+\sqrt{\left(I_{j k}^{2}-1\right) r_{k}^{2}+l_{j k}^{2}}$. Hence $r_{j}$ is uniquely determined by $r_{k}$. By the same argument $r_{i}=-I_{k i} r_{k}+\sqrt{\left(I_{k i}^{2}-1\right) r_{k}^{2}+l_{k i}^{2}}$. 
After substituting $r_{i}, r_{j}$ in the third equation, we need to show it has a unique solution for $r_{k}$. Consider the function

$$
f(t)=r_{i}^{2}(t)+r_{j}^{2}(t)+2 I_{i j} r_{i}(t) r_{j}(t)-l_{i j}^{2}
$$

which is obtained by replacing $r_{k}$ by $t$ for simplicity of notation. Now

$$
f^{\prime}(t)=\left(2 r_{i}+2 I_{i j} r_{j}\right) r_{i}^{\prime}(t)+\left(2 r_{j}+2 I_{i j} r_{i}\right) r_{j}^{\prime}(t) .
$$

Also, we have

$$
r_{i}^{\prime}(t)=-I_{k i}+\frac{\left(I_{k i}^{2}-1\right) t}{\sqrt{\left(I_{k i}^{2}-1\right) t^{2}+l_{k i}^{2}}} .
$$

If $I_{k i} \in[0,1]$, then each term in $r_{i}^{\prime}(t)$ is nonpositive. Thus $r_{i}^{\prime}(t)<0$.

If $I_{k i} \in(1, \infty)$, then

$$
r_{i}^{\prime}(t)<-I_{k i}+\frac{\left(I_{k i}^{2}-1\right) t}{\sqrt{\left(I_{k i}^{2}-1\right) t^{2}}}=-I_{k i}+\sqrt{I_{k i}^{2}-1}<0 .
$$

By the same argument, we have $r_{j}^{\prime}(t)<0$. Since $2 r_{i}+2 I_{i j} r_{j}>0$ and $2 r_{j}+2 I_{i j} r_{i}>$ 0 , then $f^{\prime}(t)<0$ holds. Therefore $f(t)=0$ has a unique solution.

To determine the image $\rho_{E}\left(\mathbb{R}_{>0}^{3}\right)$, we consider the image of the boundary of $\mathbb{R}_{\geq 0}^{3}$. If $r_{i}=0$, then $r_{k}=l_{k i}, r_{j}=l_{i j}$. Thus

$$
l_{j k}^{2}=r_{j}^{2}+r_{k}^{2}+2 I_{j k} r_{j} r_{k}=l_{i j}^{2}+l_{k i}^{2}+2 I_{j k} l_{i j} l_{k i} .
$$

On the other hand, if $r_{i}>0$, then $r_{k}<l_{k i}, r_{j}<l_{i j}$. We have

$$
l_{j k}^{2}<l_{i j}^{2}+l_{k i}^{2}+2 I_{j k} l_{i j} l_{k i} .
$$

Therefore the image $\rho_{E}\left(\mathbb{R}_{>0}^{3}\right)$ is the subset of vectors $\left(l_{i j}, l_{j k}, l_{k i}\right)$ satisfying

$$
\begin{aligned}
& l_{j k}^{2}<l_{i j}^{2}+l_{k i}^{2}+2 I_{j k} l_{i j} l_{k i}, \\
& l_{k i}^{2}<l_{j k}^{2}+l_{i j}^{2}+2 I_{k i} l_{j k} l_{i j}, \\
& l_{i j}^{2}<l_{k i}^{2}+l_{j k}^{2}+2 I_{i j} l_{k i} l_{j k} .
\end{aligned}
$$

In fact, $\rho_{E}\left(\mathbb{R}_{>0}^{3}\right)$ is a cone bounded by three surfaces. In $\mathbb{R}_{\geq 0}^{3}$, the intersection of any of the two surfaces is a straight line. The three lines of intersection are

$$
\begin{aligned}
& l_{i j}=0 \text { and } l_{j k}=l_{k i}, \\
& l_{j k}=0 \text { and } l_{k i}=l_{i j}, \\
& l_{k i}=0 \text { and } l_{i j}=l_{j k} .
\end{aligned}
$$

Let $\mathcal{L}$ be the subset of $\mathbb{R}_{>0}^{3}$ formed by vectors $\left(l_{i j}, l_{j k}, l_{k i}\right)$ satisfying the triangle inequality. Therefore $\mathcal{L}$ is a cone bounded by three planes. The three lines of intersection of the three planes are the same as that of the boundary surfaces of $\rho_{E}\left(\mathbb{R}_{>0}^{3}\right)$.

We claim that $\rho_{E}\left(\mathbb{R}_{>0}^{3}\right) \cap \mathcal{L}$ is a cone. Therefore it is connected and simply connected.

In fact, if $I_{i j} \in[0,1]$, then in $\mathbb{R}_{>0}^{3}$ we have

$$
\left\{\left(l_{i j}, l_{j k}, l_{k i}\right) \mid l_{i j}^{2}<l_{k i}^{2}+l_{j k}^{2}+2 I_{i j} l_{k i} l_{j k}\right\} \subseteq\left\{\left(l_{i j}, l_{j k}, l_{k i}\right) \mid l_{i j}<l_{k i}+l_{j k}\right\} .
$$

If $I_{i j} \in(1, \infty)$, then in $\mathbb{R}_{>0}^{3}$ we have

$$
\left\{\left(l_{i j}, l_{j k}, l_{k i}\right) \mid l_{i j}^{2}<l_{k i}^{2}+l_{j k}^{2}+2 I_{i j} l_{k i} l_{j k}\right\} \supset\left\{\left(l_{i j}, l_{j k}, l_{k i}\right) \mid l_{i j}<l_{k i}+l_{j k}\right\} .
$$

To determine the shape of $\rho_{E}\left(\mathbb{R}_{>0}^{3}\right) \cap \mathcal{L}$, there are four cases to consider. 
If $I_{i j}, I_{j k}, I_{k i} \in(1, \infty)$, then $\rho_{E}\left(\mathbb{R}_{>0}^{3}\right) \cap \mathcal{L}=\mathcal{L}$.

If $I_{i j} \in[0,1], I_{j k}, I_{k i} \in(1, \infty)$, then $\rho_{E}\left(\mathbb{R}_{>0}^{3}\right) \cap \mathcal{L}$ is a cone bounded by one surface and two planes.

If $I_{i j}, I_{j k} \in[0,1], I_{k i} \in(1, \infty)$, then $\rho_{E}\left(\mathbb{R}_{>0}^{3}\right) \cap \mathcal{L}$ is a cone bounded by two surfaces and one plane.

If $I_{i j}, I_{j k}, I_{k i} \in[0,1]$, then $\rho_{E}\left(\mathbb{R}_{>0}^{3}\right) \cap \mathcal{L}=\rho_{E}\left(\mathbb{R}_{>0}^{3}\right)$.

To finish the proof of the lemma, by definition, $\mathcal{R}_{E}^{i j k}=\rho_{E}^{-1}\left(\rho_{E}\left(\mathbb{R}_{>0}^{3}\right) \cap \mathcal{L}\right)$. Since $\rho_{E}$ is a smooth embedding, $\mathcal{R}_{E}^{i j k}$ is connected and simply connected.

In the construction of the energy function, we will use a natural variable $u=$ $\left(u_{1}, u_{2}, \ldots, u_{|V|}\right)$, where $u_{i}=\ln \left(r_{i}\right)$. Let $\tau_{E}: \mathbb{R}^{3} \rightarrow \mathbb{R}_{>0}^{3}$ be the map sending $\left(u_{i}, u_{j}, u_{k}\right)$ to $\left(r_{i}, r_{j}, r_{k}\right)$. Since $\tau_{E}$ is a diffeomorphism, we have

Corollary 4. The set $\mathcal{U}_{E}^{i j k}:=\tau_{E}^{-1}\left(\mathcal{R}_{E}^{i j k}\right)$ is a connected and simply connected open subset in $\mathbb{R}^{3}$.

To prove Theorem 2, we construct a concave energy function on $\mathcal{U}_{E}^{i j k}$ for each triangle $\triangle i j k \in F$. We obtain the local rigidity of inversive distance circle packing from this energy function. We cannot prove the global rigidity due to the fact that $\mathcal{U}_{E}^{i j k}$ is not always convex in this general setting. In fact, $l_{i j}, l_{j k}, l_{k i}$ satisfying the triangle inequality is equivalent to

$$
\left(l_{i j}+l_{j k}+l_{k i}\right)\left(l_{j k}+l_{k i}-l_{i j}\right)\left(l_{j k}+l_{i j}-l_{k i}\right)\left(l_{i j}+l_{j k}-l_{k i}\right)>0 .
$$

Substituting the functions $l_{i j}, l_{j k}, l_{k i}$ in terms of $u_{i}, u_{j}, u_{k}$ into inequality (1), we see that $\mathcal{U}_{E}^{i j k}$ is defined by the inequality

$$
\begin{aligned}
\frac{1-I_{j k}^{2}}{e^{2 u_{i}}}+\frac{1-I_{k i}^{2}}{e^{2 u_{j}}} & +\frac{1-I_{i j}^{2}}{e^{2 u_{k}}} \\
& +\frac{2\left(I_{j k} I_{k i}+I_{i j}\right)}{e^{u_{i}+u_{j}}}+\frac{2\left(I_{k i} I_{i j}+I_{j k}\right)}{e^{u_{j}+u_{k}}}+\frac{2\left(I_{i j} I_{j k}+I_{k i}\right)}{e^{u_{k}+u_{i}}}>0 .
\end{aligned}
$$

For example, when $I_{i j}=I_{j k}=I_{k i}=2$, the inequality (2) determines a subset which is not convex.

In the Andreev-Thurston Theorem, the space of cone angles is determined by a continuity argument. Since we cannot prove the global rigidity, we are not able to determine the space of cone angles in the case of inversive distance circle packing.

2.2. Energy function. To prove Theorem 2, we will construct an energy function on the space of radius vectors. First, we construct an energy function on the space for one triangle. To simplify notation, consider the triangle $\triangle 123 \in F$ and the map

$$
\begin{aligned}
& \mathcal{U}_{E}^{123} \quad \rightarrow \quad \mathcal{R}_{E}^{123} \quad \rightarrow \quad \mathcal{L} \quad \rightarrow \quad \mathbb{R}_{>0}^{3}, \\
& \left(u_{1}, u_{2}, u_{3}\right) \mapsto\left(r_{1}, r_{2}, r_{3}\right) \mapsto\left(l_{1}, l_{2}, l_{3}\right) \mapsto\left(\alpha_{1}, \alpha_{2}, \alpha_{3}\right),
\end{aligned}
$$

where $r_{i}=e^{u_{i}}$ for $i=1,2,3$. For three fixed positive numbers $I_{12}, I_{23}, I_{31}$, we have $l_{i}^{2}=r_{j}^{2}+r_{k}^{2}+2 I_{j k} r_{j} r_{k}$ for $\{i, j, k\}=\{1,2,3\}$. For simplicity of notation, we set $\left(l_{1}, l_{2}, l_{3}\right)=\left(l_{23}, l_{31}, l_{12}\right)$. Also, $\alpha_{1}, \alpha_{2}, \alpha_{3}$ are inner angles of a Euclidean triangle with edge lengths $l_{1}, l_{2}, l_{3}$. By the cosine law, $\cos \alpha_{i}=\frac{-l_{i}^{2}+l_{j}^{2}+l_{k}^{2}}{2 l_{j} l_{k}}$ for $\{i, j, k\}=$ $\{1,2,3\}$. Therefore $\alpha_{1}, \alpha_{2}, \alpha_{3}$ are functions in terms of $u_{1}, u_{2}, u_{3}$.

Lemma 5. The Jacobian matrix of functions $\alpha_{1}, \alpha_{2}, \alpha_{3}$ in terms of $u_{1}, u_{2}, u_{3}$ is symmetric. 
Proof. Due to the cosine law of a Euclidean triangle, $\alpha_{1}, \alpha_{2}, \alpha_{3}$ are functions of $l_{1}, l_{2}, l_{3}$. The following formula can be proved by direct calculation. For $\{i, j, k\}=$ $\{1,2,3\}$,

$$
\left(\begin{array}{l}
d \alpha_{1} \\
d \alpha_{2} \\
d \alpha_{3}
\end{array}\right)=\frac{-1}{\sin \alpha_{i} l_{j} l_{k}}\left(\begin{array}{ccc}
l_{1} & 0 & 0 \\
0 & l_{2} & 0 \\
0 & 0 & l_{3}
\end{array}\right)\left(\begin{array}{ccc}
-1 & \cos \alpha_{3} & \cos \alpha_{2} \\
\cos \alpha_{3} & -1 & \cos \alpha_{1} \\
\cos \alpha_{2} & \cos \alpha_{1} & -1
\end{array}\right)\left(\begin{array}{l}
d l_{1} \\
d l_{2} \\
d l_{3}
\end{array}\right)
$$

Next, by the construction $l_{i}^{2}=r_{j}^{2}+r_{k}^{2}+2 I_{j k} r_{j} r_{k}$, for $\{i, j, k\}=\{1,2,3\}$, where $I_{j k}$ is a constant. Differentiating the two sides of the equality, we obtain

$$
d l_{i}=\frac{l_{i}^{2}+r_{j}^{2}-r_{k}^{2}}{2 l_{i} r_{j}} d r_{j}+\frac{l_{i}^{2}+r_{k}^{2}-r_{j}^{2}}{2 l_{i} r_{k}} d r_{k}
$$

Finally, $r_{i}=e^{u_{i}}$ implies $r_{i} d u_{i}=d r_{i}$, for $i=1,2,3$.

Combining the three relations, we have, for $\{i, j, k\}=\{1,2,3\}$,

$$
\begin{aligned}
& \left(\begin{array}{l}
d \alpha_{1} \\
d \alpha_{2} \\
d \alpha_{3}
\end{array}\right)=\frac{-1}{\sin \alpha_{i} l_{j} l_{k}}\left(\begin{array}{ccc}
l_{1} & 0 & 0 \\
0 & l_{2} & 0 \\
0 & 0 & l_{3}
\end{array}\right)\left(\begin{array}{ccc}
-1 & \cos \alpha_{3} & \cos \alpha_{2} \\
\cos \alpha_{3} & -1 & \cos \alpha_{1} \\
\cos \alpha_{2} & \cos \alpha_{1} & -1
\end{array}\right) \\
& \times\left(\begin{array}{ccc}
0 & \frac{l_{1}^{2}+r_{2}^{2}-r_{3}^{2}}{2 l_{1} r_{2}} & \frac{l_{1}^{2}+r_{3}^{2}-r_{2}^{2}}{2 l_{1} r_{3}} \\
\frac{l_{2}^{2}+r_{1}^{2}-r_{3}^{2}}{2 l_{2} r_{1}} & 0 & \frac{l_{2}^{2}+r_{3}^{2}-r_{1}^{2}}{2 l_{2} r_{3}} \\
\frac{l_{3}^{2}+r_{1}^{2}-r_{2}^{2}}{2 l_{3} r_{1}} & \frac{l_{3}^{2}+r_{2}^{2}-r_{1}^{2}}{2 l_{3} r_{2}} & 0
\end{array}\right)\left(\begin{array}{ccc}
r_{1} & 0 & 0 \\
0 & r_{2} & 0 \\
0 & 0 & r_{3}
\end{array}\right)\left(\begin{array}{l}
d u_{1} \\
d u_{2} \\
d u_{3}
\end{array}\right) .
\end{aligned}
$$

We write the above formula as

$$
\left(\begin{array}{c}
d \alpha_{1} \\
d \alpha_{2} \\
d \alpha_{3}
\end{array}\right)=\frac{-1}{\sin \alpha_{i} l_{j} l_{k}} N\left(\begin{array}{c}
d u_{1} \\
d u_{2} \\
d u_{3}
\end{array}\right)
$$

To show that the Jacobian matrix of functions $\alpha_{1}, \alpha_{2}, \alpha_{3}$ in terms of $u_{1}, u_{2}, u_{3}$ is symmetric is equivalent to showing that the matrix $N$ is symmetric.

By the cosine law, we have

$$
\begin{array}{r}
4 N=\left(\begin{array}{ccc}
-2 l_{1}^{2} & l_{1}^{2}+l_{2}^{2}-l_{3}^{2} & l_{3}^{2}+l_{1}^{2}-l_{2}^{2} \\
l_{1}^{2}+l_{2}^{2}-l_{3}^{2} & -2 l_{2}^{2} & l_{2}^{2}+l_{3}^{2}-l_{1}^{2} \\
l_{3}^{2}+l_{1}^{2}-l_{2}^{2} & l_{2}^{2}+l_{3}^{2}-l_{1}^{2} & -2 l_{3}^{2}
\end{array}\right)\left(\begin{array}{ccc}
\frac{1}{l_{1}^{2}} & 0 & 0 \\
0 & \frac{1}{l_{2}^{2}} & 0 \\
0 & 0 & \frac{1}{l_{3}^{2}}
\end{array}\right) \\
\times\left(\begin{array}{ccc}
0 & l_{1}^{2}+r_{2}^{2}-r_{3}^{2} & l_{1}^{2}+r_{3}^{2}-r_{2}^{2} \\
l_{2}^{2}+r_{1}^{2}-r_{3}^{2} & 0 & l_{2}^{2}+r_{3}^{2}-r_{1}^{2} \\
l_{3}^{2}+r_{1}^{2}-r_{2}^{2} & l_{3}^{2}+r_{2}^{2}-r_{1}^{2} & 0
\end{array}\right) .
\end{array}
$$


To simplify notation, let $a:=l_{1}^{2}, b:=l_{2}^{2}, c:=l_{3}^{2}, x:=\frac{r_{2}^{2}-r_{3}^{2}}{a}, y:=\frac{r_{3}^{2}-r_{1}^{2}}{b}, z:=$ $\frac{r_{1}^{2}-r_{2}^{2}}{c}$. Note that $a x+b y+c z=0$. Thus

$$
\begin{aligned}
4 N= & \left(\begin{array}{ccc}
-2 a & a+b-c & c+a-b \\
a+b-c & -2 b & b+c-a \\
c+a-b & b+c-a & -2 c
\end{array}\right)\left(\begin{array}{ccc}
0 & 1+x & 1-x \\
1-y & 0 & 1+y \\
1+z & 1-z & 0
\end{array}\right) \\
& =\left(\begin{array}{ccc}
2 a-(a+b-c) y+(c+a-b) z & c-a-b-2 a x-(c+a-b) z & b-a-c+2 a x+(a+b-c) y \\
c-a-b+2 b y+(b+c-a) z & 2 b-(b+c-a) z+(a+b-c) x & a-b-c-2 b y-(a+b-c) x \\
b-c-a-2 c z-(b+c-a) y & a-b-c+2 c z+(c+a-b) x & 2 c-(c+a-b) x+(b+c-a) y
\end{array}\right) .
\end{aligned}
$$

This matrix is symmetric due to the equality $a x+b y+c z=0$.

Glickenstein [11] gave a new proof of the above lemma. The new proof puts the result in a general context and reveals its geometric meaning.

Lemma 6. The Jacobian matrix of functions $\alpha_{1}, \alpha_{2}, \alpha_{3}$ in terms of $u_{1}, u_{2}, u_{3}$ has one zero eigenvalue with associated eigenvector $(1,1,1)$ and two negative eigenvalues.

Proof. Using the notation in Lemma 5, we need to show that the matrix $4 N$ has one zero eigenvalue and two positive eigenvalues. Since $4 N$ is symmetric and the sum of entries in each row is zero, we can write $4 N$ as

$$
\left(\begin{array}{ccc}
-B_{2}-B_{3} & B_{3} & B_{2} \\
B_{3} & -B_{3}-B_{1} & B_{1} \\
B_{2} & B_{1} & -B_{1}-B_{2}
\end{array}\right)
$$

where $B_{1}, B_{2}, B_{3}$ are functions of $a, b, c, x, y, z$.

The characteristic equation of $4 N$ is

$$
\lambda\left(\lambda^{2}+2\left(B_{1}+B_{2}+B_{3}\right) \lambda+3\left(B_{1} B_{2}+B_{2} B_{3}+B_{3} B_{1}\right)\right)=0 .
$$

There is a zero eigenvalue, and the associated eigenvector is $(1,1,1)$. We claim that $B_{1}+B_{2}+B_{3}<0$ and $B_{1} B_{2}+B_{2} B_{3}+B_{3} B_{1}>0$. These two inequalities imply that the matrix $4 N$ has two positive eigenvalues. In the following we verify the two inequalities.

First,

$$
\begin{aligned}
& B_{1}+B_{2}+B_{3}<0 \\
\Longleftrightarrow & \text { trace of } 4 N=-2\left(B_{1}+B_{2}+B_{3}\right)>0 \\
\Longleftrightarrow & a+b+c+(b-c) x+(c-a) y+(a-b) z>0 \\
\Longleftrightarrow & l_{1}^{2}+l_{2}^{2}+l_{3}^{2}+\left(l_{2}^{2}-l_{3}^{2}\right) \frac{r_{2}^{2}-r_{3}^{2}}{l_{1}^{2}}+\left(l_{3}^{2}-l_{1}^{2}\right) \frac{r_{3}^{2}-r_{1}^{2}}{l_{2}^{2}}+\left(l_{1}^{2}-l_{2}^{2}\right) \frac{r_{1}^{2}-r_{2}^{2}}{l_{3}^{2}}>0 \\
\Longleftrightarrow & l_{1}^{2}+l_{2}^{2}+l_{3}^{2} \\
& +\frac{l_{1}^{2} l_{2}^{2}+l_{1}^{2} l_{3}^{2}-l_{2}^{4}-l_{3}^{4}}{l_{2}^{2} l_{3}^{2}} r_{1}^{2}+\frac{l_{2}^{2} l_{1}^{2}+l_{2}^{2} l_{3}^{2}-l_{1}^{4}-l_{3}^{4}}{l_{1}^{2} l_{3}^{2}} r_{2}^{2}+\frac{l_{3}^{2} l_{1}^{2}+l_{3}^{2} l_{2}^{2}-l_{1}^{4}-l_{2}^{4}}{l_{1}^{2} l_{2}^{2}} r_{3}^{2}>0 \\
\Longleftrightarrow & l_{1}^{2}+l_{2}^{2}+l_{3}^{2}+\Xi_{1} r_{1}^{2}+\Xi_{2} r_{2}^{2}+\Xi_{3} r_{3}^{2}>0 .
\end{aligned}
$$

For $\{i, j, k\}=\{1,2,3\}$, since $l_{i}^{2}=r_{j}^{2}+r_{k}^{2}+2 I_{j k} r_{j} r_{k}$ and $I_{i j} \in[0, \infty)$, we have $l_{i}>r_{j}$.

Without loss of generality, we may assume $l_{1} \geq l_{2} \geq l_{3}$. Therefore $\Xi_{1} \geq 0$ and $\Xi_{3} \leq 0$ 
Case 1. If $\Xi_{2} \geq 0$, then

$$
\begin{aligned}
& l_{1}^{2}+l_{2}^{2}+l_{3}^{2}+\Xi_{1} r_{1}^{2}+\Xi_{2} r_{2}^{2}+\Xi_{3} r_{3}^{2} \\
& \geq l_{1}^{2}+l_{2}^{2}+l_{3}^{2}+\Xi_{3} r_{3}^{2} \\
& >l_{1}^{2}+l_{2}^{2}+l_{3}^{2}+\Xi_{3} l_{2}^{2} \\
& =l_{1}^{2}+l_{2}^{2}+l_{3}^{2}+\frac{l_{3}^{2} l_{1}^{2}+l_{3}^{2} l_{2}^{2}-l_{1}^{4}-l_{2}^{4}}{l_{1}^{2} l_{2}^{2}} l_{2}^{2} \\
& =\frac{1}{l_{1}^{2}}\left(\left(l_{1}^{2}-l_{2}^{2}\right) l_{2}^{2}+2 l_{1}^{2} l_{3}^{2}+l_{2}^{2} l_{3}^{2}\right)>0
\end{aligned}
$$

due to the assumption $l_{1} \geq l_{2}$.

Case 2. If $\Xi_{2}<0$, then

$$
\begin{aligned}
& l_{1}^{2}+l_{2}^{2}+l_{3}^{2}+\Xi_{1} r_{1}^{2}+\Xi_{2} r_{2}^{2}+\Xi_{3} r_{3}^{2} \\
& >l_{1}^{2}+l_{2}^{2}+l_{3}^{2}+\Xi_{2} r_{2}^{2}+\Xi_{3} r_{3}^{2} \\
& >l_{1}^{2}+l_{2}^{2}+l_{3}^{2}+\Xi_{2} l_{3}^{2}+\Xi_{3} l_{2}^{2} \\
& =l_{1}^{2}+l_{2}^{2}+l_{3}^{2}+\frac{l_{2}^{2} l_{1}^{2}+l_{2}^{2} l_{3}^{2}-l_{1}^{4}-l_{3}^{4}}{l_{1}^{2} l_{3}^{2}} l_{3}^{2}+\frac{l_{3}^{2} l_{1}^{2}+l_{3}^{2} l_{2}^{2}-l_{1}^{4}-l_{2}^{4}}{l_{1}^{2} l_{2}^{2}} l_{2}^{2} \\
& =\frac{1}{l_{1}^{2}}\left(2 l_{1}^{2} l_{2}^{2}+2 l_{2}^{2} l_{3}^{2}+2 l_{3}^{2} l_{1}^{2}-l_{1}^{4}-l_{2}^{4}-l_{3}^{4}\right) \\
& =\frac{1}{l_{1}^{2}}\left(l_{1}+l_{2}+l_{3}\right)\left(l_{1}+l_{2}-l_{3}\right)\left(l_{3}+l_{1}-l_{2}\right)\left(l_{2}+l_{3}-l_{1}\right)>0
\end{aligned}
$$

due to the triangle inequality.

Thus $B_{1}+B_{2}+B_{3}<0$ holds.

To prove $B_{1} B_{2}+B_{2} B_{3}+B_{3} B_{1}>0$, we substitute

$$
\begin{aligned}
& B_{1}=4 N_{23}=a-b-c-2 b y-(a+b-c) x, \\
& B_{2}=4 N_{31}=b-c-a-2 c z-(b+c-a) y, \\
& B_{3}=4 N_{12}=c-a-b-2 a x-(c+a-b) z
\end{aligned}
$$

to obtain

$$
\begin{aligned}
& B_{1} B_{2}+B_{2} B_{3}+B_{3} B_{1} \\
= & 2 a b+2 b c+2 a c-a^{2}-b^{2}-c^{2} \\
& +\left(b^{2}-c^{2}-3 a^{2}+6 a b+4 a c\right) x y \\
& +\left(c^{2}-a^{2}-3 b^{2}+6 b c+4 a b\right) y z \\
& +\left(a^{2}-b^{2}-3 c^{2}+6 a c+4 b c\right) z x \\
& +2 a(a+b-c) x^{2}+2 b(b+c-a) y^{2}+2 c(c+a-b) z^{2} \\
& +2(a+b+c)(a x+b y+c z) \\
= & \left(2 a b+2 b c+2 a c-a^{2}-b^{2}-c^{2}\right)(x y+y z+z x+1) \\
& +2(a x+b y+c z)((a+b-c) x+(b+c-a) y+(c+a-b) z) \\
& +2(a+b+c)(a x+b y+c z) \\
= & \left(2 a b+2 b c+2 a c-a^{2}-b^{2}-c^{2}\right)(x y+y z+z x+1)
\end{aligned}
$$

due to the fact that $a x+b y+c z=0$. 
Since

$$
\begin{aligned}
& 2 a b+2 b c+2 a c-a^{2}-b^{2}-c^{2} \\
= & 2 l_{1}^{2} l_{2}^{2}+2 l_{2}^{2} l_{3}^{2}+2 l_{3}^{2} l_{1}^{2}-l_{1}^{4}-l_{2}^{4}-l_{3}^{4} \\
= & \left(l_{1}+l_{2}+l_{3}\right)\left(l_{1}+l_{2}-l_{3}\right)\left(l_{3}+l_{1}-l_{2}\right)\left(l_{2}+l_{3}-l_{1}\right)>0,
\end{aligned}
$$

we have

$$
\begin{aligned}
& B_{1} B_{2}+B_{2} B_{3}+B_{3} B_{1}>0 \\
\Longleftrightarrow & x y+y z+z x+1>0
\end{aligned}
$$

$$
\Longleftrightarrow\left(r_{1}^{2}-r_{2}^{2}\right)\left(r_{3}^{2}-r_{1}^{2}\right) l_{1}^{2}+\left(r_{2}^{2}-r_{3}^{2}\right)\left(r_{1}^{2}-r_{2}^{2}\right) l_{2}^{2}+\left(r_{3}^{2}-r_{1}^{2}\right)\left(r_{2}^{2}-r_{3}^{2}\right) l_{3}^{2}+l_{1}^{2} l_{2}^{2} l_{3}^{2}>0 .
$$

To verify inequality (3), first note that, since $l_{i}^{2}=r_{j}^{2}+r_{k}^{2}+2 I_{j k} r_{j} r_{k}$ and $I_{j k} \in$ $[0, \infty)$, we have $l_{i}^{2} \geq r_{j}^{2}+r_{k}^{2}$ for $\{i, j, k\}=\{1,2,3\}$.

Since the coefficient of $l_{1}^{2}$ on the left hand side of (3) satisfies

$$
\begin{aligned}
\left(r_{1}^{2}-r_{2}^{2}\right)\left(r_{3}^{2}-r_{1}^{2}\right)+l_{2}^{2} l_{3}^{2} & \geq\left(r_{1}^{2}-r_{2}^{2}\right)\left(r_{3}^{2}-r_{1}^{2}\right)+\left(r_{1}^{2}+r_{3}^{2}\right)\left(r_{1}^{2}+r_{2}^{2}\right) \\
& =2 r_{1}^{2} r_{2}^{2}+2 r_{1}^{2} r_{3}^{2}>0,
\end{aligned}
$$

by replacing $l_{1}^{2}$ by $r_{2}^{2}+r_{3}^{2}$, we see that the left hand side of (3) is not less than

$$
\begin{aligned}
\left(r_{1}^{2}-r_{2}^{2}\right)\left(r_{3}^{2}-r_{1}^{2}\right)\left(r_{2}^{2}+r_{3}^{2}\right)+\left(r_{2}^{2}-r_{3}^{2}\right)\left(r_{1}^{2}-r_{2}^{2}\right) l_{2}^{2}+\left(r_{3}^{2}-r_{1}^{2}\right) & \left(r_{2}^{2}-r_{3}^{2}\right) l_{3}^{2} \\
& +\left(r_{2}^{2}+r_{3}^{2}\right) l_{2}^{2} l_{3}^{2} .
\end{aligned}
$$

Since the coefficient of $l_{2}^{2}$ in (4) satisfies

$$
\begin{aligned}
\left(r_{2}^{2}-r_{3}^{2}\right)\left(r_{1}^{2}-r_{2}^{2}\right)+\left(r_{2}^{2}+r_{3}^{2}\right) l_{3}^{2} & \geq\left(r_{2}^{2}-r_{3}^{2}\right)\left(r_{1}^{2}-r_{2}^{2}\right)+\left(r_{2}^{2}+r_{3}^{2}\right)\left(r_{1}^{2}+r_{2}^{2}\right) \\
& =2 r_{1}^{2} r_{2}^{2}+2 r_{2}^{2} r_{3}^{2}>0,
\end{aligned}
$$

by replacing $l_{2}^{2}$ by $r_{1}^{2}+r_{3}^{2}$, we see that (4) is not less than

$$
\begin{aligned}
\left(r_{1}^{2}-r_{2}^{2}\right)\left(r_{3}^{2}-r_{1}^{2}\right)\left(r_{2}^{2}+r_{3}^{2}\right)+ & \left(r_{2}^{2}-r_{3}^{2}\right)\left(r_{1}^{2}-r_{2}^{2}\right)\left(r_{1}^{2}+r_{3}^{2}\right) \\
& +\left(r_{3}^{2}-r_{1}^{2}\right)\left(r_{2}^{2}-r_{3}^{2}\right) l_{3}^{2}+\left(r_{2}^{2}+r_{3}^{2}\right)\left(r_{1}^{2}+r_{3}^{2}\right) l_{3}^{2} .
\end{aligned}
$$

Since the coefficient of $l_{3}^{2}$ in (8) is positive, by replacing $l_{3}^{2}$ by $r_{1}^{2}+r_{2}^{2}$, we see that (5D) is not less than

$$
\begin{aligned}
\left(r_{1}^{2}-r_{2}^{2}\right)\left(r_{3}^{2}-r_{1}^{2}\right) & \left(r_{2}^{2}+r_{3}^{2}\right)+\left(r_{2}^{2}-r_{3}^{2}\right)\left(r_{1}^{2}-r_{2}^{2}\right)\left(r_{1}^{2}+r_{3}^{2}\right) \\
& +\left(r_{3}^{2}-r_{1}^{2}\right)\left(r_{2}^{2}-r_{3}^{2}\right)\left(r_{1}^{2}+r_{2}^{2}\right)+\left(r_{2}^{2}+r_{3}^{2}\right)\left(r_{1}^{2}+r_{3}^{2}\right)\left(r_{1}^{2}+r_{2}^{2}\right) .
\end{aligned}
$$

Since (66) is equal to $8 r_{1}^{2} r_{2}^{2} r_{3}^{2}$, we see that $B_{1} B_{2}+B_{2} B_{3}+B_{3} B_{1}>0$.

For the special case of intersection angle circle packing, Lemma 6 was proved in Chow-Luo [9] (Lemma 3.1). In that case, by a geometric reasoning due to Thurston 23. the angle $\alpha_{i}$ is a decreasing function of $r_{i}$ and an increasing function of $r_{j}$ for $j \neq i$. That means $\frac{\partial \alpha_{i}}{\partial r_{i}}<0$ and $\frac{\partial \alpha_{i}}{\partial r_{j}}>0$ for $j \neq i$. Therefore the diagonal entries of the matrix $N$ in Lemma 5 are positive and the off-diagonal entries of $N$ are negative. By a theorem in linear algebra, this property implies Lemma 6. But Chow-Luo's proof does not work in the general case of inversive distance circle packing, because it is not always true that the angle $\alpha_{i}$ is a decreasing function of $r_{i}$ and an increasing 
function of $r_{j}$ for $j \neq i$. For example, when $l_{1}=2, l_{2}=2, l_{3}=3, r_{1}=r_{2}=r_{3}=1$, the matrix $N$ is

$$
\left(\begin{array}{ccc}
2 & \frac{1}{4} & -\frac{9}{4} \\
\frac{1}{4} & 2 & -\frac{9}{4} \\
-\frac{9}{4} & -\frac{9}{4} & \frac{9}{2}
\end{array}\right)
$$

with eigenvalue $0, \frac{27}{4}, \frac{7}{4}$. This shows that $\frac{\partial \alpha_{1}}{\partial r_{2}}<0$, while $\frac{\partial \alpha_{1}}{\partial r_{3}}>0$ for some value of $l_{i}$ 's and $r_{i}$ 's.

Since, by Corollary 4 , the space $\mathcal{U}_{E}^{123}$ of vectors $\left(u_{1}, u_{2}, u_{3}\right)$ is connected and simply connected, Lemma 5 and Lemma 6 imply

Corollary 7. The differential 1-form $\sum_{i=1}^{3} \alpha_{i} d u_{i}$ is closed. For any $c \in \mathcal{U}_{E}^{123}$, the integration $w\left(u_{1}, u_{2}, u_{3}\right)=\int_{c}^{\left(u_{1}, u_{2}, u_{3}\right)} \sum_{i=1}^{3} \alpha_{i} d u_{i}$ is a concave function on $\mathcal{U}_{E}^{123}$ and satisfies, for $i=1,2,3$,

$$
\frac{\partial w}{\partial u_{i}}=\alpha_{i}
$$

Proof of Theorem 2 in Euclidean geometry. Let's prove the local rigidity of inversive distance circle packing in Euclidean geometry. For a weighted triangulated closed surface $(\Sigma, T, I)$ with $I_{i j} \in[0, \infty)$ for each $i j \in E$, let $\mathcal{U}_{E}$ be the open subset of $\mathbb{R}^{|V|}$ formed by the vectors $u=\left(u_{1}, u_{2}, \ldots, u_{|V|}\right)$ satisfying $\left(u_{i}, u_{j}, u_{k}\right) \in \mathcal{U}_{E}^{i j k}$ whenever $\triangle i j k \in F$.

For a radius vector $r=\left(r_{1}, r_{2}, \ldots, r_{|V|}\right)$, we may rescale it to get $c r$, while the cone angles remain the same. Under the rescaling, $u$ changes to $u+\ln (c)(1,1, \ldots, 1)$. Therefore we only consider the intersection $\mathcal{U}_{E} \cap P$ where $P$ is the hyperplane defined by $\sum_{i=1}^{|V|} u_{i}=0$.

Let $a_{i}$ be the cone angle at vertex $i$ under the Euclidean cone metric. Then $\sum_{i=1}^{|V|} a_{i}=$ the sum of all inner angles $=\pi|F|$. Therefore the space of possible cone angles is contained in the intersection $\mathbb{R}_{>0}^{|V|} \cap Q$, where $Q$ is the hyperplane defined by $\sum_{i=1}^{|V|} a_{i}=\pi|F|$. We have a map $\xi: \mathcal{U}_{E} \cap P \rightarrow \mathbb{R}_{>0}^{|V|} \cap Q$.

By Corollary 7 for each triangle $\triangle i j k \in F$ there is a function $w\left(u_{i}, u_{j}, u_{k}\right)$. Define a function $W: \mathcal{U}_{E} \rightarrow \mathbb{R}$ by

$$
W\left(u_{1}, u_{2}, \ldots, u_{|V|}\right)=\sum_{\triangle i j k \in F} w\left(u_{i}, u_{j}, u_{k}\right),
$$

where the sum is over all triangles in $F$.

By (7), $\frac{\partial W}{\partial u_{i}}$ equals the sum of inner angles having vertex $i$, i.e., the cone angle at vertex $i$. Therefore, when restricted on $\mathcal{U}_{E} \cap P$, the gradient $\nabla W$ is the map $\xi: \mathcal{U}_{E} \cap P \rightarrow \mathbb{R}_{>0}^{|V|} \cap Q$.

We claim that the restriction of $W$ on $\mathcal{U}_{E} \cap P$ is strictly concave. By the definition of $W$, we get the relation between the Hessian matrixes

$$
H(W)=\sum_{\triangle i j k \in F} H\left(w\left(u_{i}, u_{j}, u_{k}\right)\right)
$$

where we think of $w\left(u_{i}, u_{j}, u_{k}\right)$ as a function on $\mathcal{U}_{E}$. 
For any $x \in \mathcal{U}_{E} \cap P$,

$$
\begin{aligned}
x H(W) x^{T} & =\sum_{\triangle i j k \in F} x H\left(w\left(u_{i}, u_{j}, u_{k}\right)\right) x^{T} \\
& =\sum_{\triangle i j k \in F}\left(x_{i}, x_{j}, x_{k}\right) H\left(w\left(u_{i}, u_{j}, u_{k}\right)\right)\left(x_{i}, x_{j}, x_{k}\right)^{T} \leq 0,
\end{aligned}
$$

since $H\left(w\left(u_{i}, u_{j}, u_{k}\right)\right)$ is negative semi-definite for each $\triangle i j k \in F$.

Thus $x H(W) x^{T}=0$ implies $\left(x_{i}, x_{j}, x_{k}\right) H\left(w\left(u_{i}, u_{j}, u_{k}\right)\right)\left(x_{i}, x_{j}, x_{k}\right)^{T}=0$ for each $\triangle i j k$. By Lemma 6. $\left(x_{i}, x_{j}, x_{k}\right)$ is an eigenvector of zero eigenvalue. Therefore $\left(x_{i}, x_{j}, x_{k}\right)=c_{i j k}(1,1,1)$ for some constant $c_{i j k}$. If two triangles share a vertex, then the corresponding constants $c_{i j k}$ of the two triangles must be the same. In fact, consider the two triangles $\triangle i j k$ and $\triangle i j^{\prime} k^{\prime}$ which share the vertex $i$. For $\triangle i j k$, we have $\left(x_{i}, x_{j}, x_{k}\right)=c_{i j k}(1,1,1)$; in particular, $x_{i}=c_{i j k}$. Also, for $\triangle i j^{\prime} k^{\prime}$, we have $\left(x_{i}, x_{j^{\prime}}, x_{k^{\prime}}\right)=c_{i j^{\prime} k^{\prime}}(1,1,1)$; in particular, $x_{i}=c_{i j^{\prime} k^{\prime}}$. Hence $c_{i j k}=x_{i}=c_{i j^{\prime} k^{\prime}}$. Since the surface is connected, the corresponding constants $c_{i j k}$ of any triangles are the same. Thus $c_{i j k}$ must be a constant $c$ independent of the triangle $\triangle i j k$. Therefore $x=c(1,1, \ldots, 1)$. Since $x \in P$, i.e, $\sum x_{i}=0$, we have $c=0$. Thus $x=(0,0, \ldots, 0)$. This shows that the Hessian $H(W)$ is negative definite when restricted on $\mathcal{U}_{E} \cap P$.

The local rigidity of inversive distance circle packing in Euclidean geometry follows from the following lemma in analysis

Lemma 8. If $X$ is an open set in $\mathbb{R}^{n}$ and the Hessian matrix of $f$ is positive definite for all $x \in X$, then the gradient $\nabla f: X \rightarrow \mathbb{R}^{n}$ is a local diffeomorphism.

\section{Circle PACKing in hyperbolic GeOMetry}

3.1. Space of radius vectors. As stated in the Introduction, to obtain a hyperbolic cone metric, we need conditions on a radius vector. In this section we investigate the space of radius vectors for one triangle in hyperbolic geometry. Consider a topological triangle $\triangle i j k \in F$. Assign a number $l_{i j}$ to the edge $i j \in E$, where $l_{i j}$ is the distance of the centers of two circles with radii $r_{i}, r_{j}$ and inversive distance $I_{i j}$. Therefore $l_{i j}$ is a function of $r_{i}, r_{j}$. In fact, in hyperbolic geometry, $l_{i j}$ is determined by $\cosh l_{i j}=\cosh r_{i} \cosh r_{j}+I_{i j} \sinh r_{i} \sinh r_{j}$. Similarly, we obtain $l_{j k}, l_{k i}$.

Lemma 9. The set $\mathcal{R}_{H}^{i j k}:=\left\{\left(r_{i}, r_{j}, r_{k}\right) \in \mathbb{R}_{>0}^{3} \mid l_{i j}, l_{j k}, l_{k i}\right.$ satisfy triangle inequal$i t y\}$ is a connected and simply connected open subset in $\mathbb{R}_{>0}^{3}$.

Proof. Let $\rho_{H}: \mathbb{R}_{>0}^{3} \rightarrow \mathbb{R}_{>0}^{3}$ be the map sending $\left(r_{i}, r_{j}, r_{k}\right)$ to $\left(l_{i j}, l_{j k}, l_{k i}\right)$. We claim that $\rho_{H}$ is a smooth embedding. First the determinant of the Jacobian matrix of $\rho_{H}$ is nonzero. Next, we will show $\rho_{H}$ is one-to-one.

That means, for fixed numbers $I_{i j}, I_{j k}, I_{k i} \in[0, \infty)$ and a fixed hyperbolic triangle, there is at most one configuration of three circles centered at the vertices of the triangle and having the prescribed inversive distance $I_{i j}, I_{j k}, I_{k i}$.

The statement is true in Euclidean geometry as proved in the proof of Lemma 3. We use the result in Euclidean geometry to prove a similar result in hyperbolic geometry. Let's assume there are two configurations of three circles in a hyperbolic plane with the fixed triangle and inversive distance. As in Figure 2, the stereographic projection sends the two configurations to Euclidean plane $\mathbb{C}$. Since the 
stereographic projection preserves the inversive distance, there will be two different configurations of three circles with the same Euclidean triangle and the same inversive distance. This is impossible.

To determine the image $\rho_{H}\left(\mathbb{R}_{>0}^{3}\right)$, we consider the image of the boundary of $\mathbb{R}_{\geq 0}^{3}$. If $r_{i}=0$, then $r_{k}=l_{k i}, r_{j}=l_{i j}$. Thus

$\cosh l_{j k}=\cosh r_{j} \cosh r_{k}+I_{j k} \sinh r_{j} \sinh r_{k}=\cosh l_{i j} \cosh l_{k i}+I_{j k} \sinh l_{i j} \sinh l_{k i}$.

On the other hand, if $r_{i}>0$, then $r_{k}<l_{k i}, r_{j}<l_{i j}$. We have

$$
\cosh l_{j k}<\cosh l_{i j} \cosh l_{k i}+I_{j k} \sinh l_{i j} \sinh l_{k i} \text {. }
$$

Therefore the image $\rho_{H}\left(\mathbb{R}_{>0}^{3}\right)$ is the subset of vectors $\left(l_{i j}, l_{j k}, l_{k i}\right)$ satisfying

$$
\begin{aligned}
& \cosh l_{j k}<\cosh l_{i j} \cosh l_{k i}+I_{j k} \sinh l_{i j} \sinh l_{k i}, \\
& \cosh l_{k i}<\cosh l_{j k} \cosh l_{i j}+I_{k i} \sinh l_{j k} \sinh l_{i j}, \\
& \cosh l_{i j}<\cosh l_{k i} \cosh l_{j k}+I_{i j} \sinh l_{k i} \sinh l_{j k} .
\end{aligned}
$$

In fact, $\rho_{H}\left(\mathbb{R}_{>0}^{3}\right)$ is a cone bounded by three surfaces. In $\mathbb{R}_{\geq 0}^{3}$, the intersection of any of the two surfaces is a straight line. For example, if

$$
\cosh l_{j k}=\cosh l_{i j} \cosh l_{k i}+I_{j k} \sinh l_{i j} \sinh l_{k i}
$$

and

$$
\cosh l_{k i}=\cosh l_{j k} \cosh l_{i j}+I_{k i} \sinh l_{j k} \sinh l_{i j},
$$

then the sum of the two equation gives

$$
\begin{aligned}
0 & =\left(\cosh l_{k i}+\cosh l_{j k}\right)\left(\cosh l_{i j}-1\right)+\left(I_{j k} \sinh l_{k i}+I_{k i} \sinh l_{j k}\right) \sinh l_{i j} \\
& =\left(\cosh l_{k i}+\cosh l_{j k}\right) 2 \sinh ^{2} \frac{l_{i j}}{2}+\left(I_{j k} \sinh l_{k i}+I_{k i} \sinh l_{j k}\right) 2 \cosh \frac{l_{i j}}{2} \sinh \frac{l_{i j}}{2}
\end{aligned}
$$

The only possibility is $\sinh \frac{l_{i j}}{2}=0$. Therefore $l_{i j}=0$ and $l_{j k}=l_{k i}$.

Hence the three lines of intersection are

$$
\begin{aligned}
& l_{i j}=0 \text { and } l_{j k}=l_{k i}, \\
& l_{j k}=0 \text { and } l_{k i}=l_{i j}, \\
& l_{k i}=0 \text { and } l_{i j}=l_{j k} .
\end{aligned}
$$

Recall that $\mathcal{L}$ is the subset of $\mathbb{R}_{>0}^{3}$ formed by vectors $\left(l_{i j}, l_{j k}, l_{k i}\right)$ satisfying the triangle inequality. Therefore $\mathcal{L}$ is a cone bounded by three planes. The three lines of intersection of the three planes are the same as that of the boundary surfaces of $\rho_{H}\left(\mathbb{R}_{>0}^{3}\right)$.

We claim that $\rho_{H}\left(\mathbb{R}_{>0}^{3}\right) \cap \mathcal{L}$ is a cone.

In fact, if $I_{i j} \in[0,1]$, then in $\mathbb{R}_{>0}^{3}$ we have

$$
\begin{aligned}
\left\{\left(l_{i j}, l_{j k}, l_{k i}\right) \mid \cosh l_{i j}<\cosh l_{k i} \cosh l_{j k}+I_{i j} \sinh l_{k i} \sinh l_{j k}\right\} & \\
& \subseteq\left\{\left(l_{i j}, l_{j k}, l_{k i}\right) \mid l_{i j}<l_{k i}+l_{j k}\right\} .
\end{aligned}
$$

If $I_{i j} \in(1, \infty)$, then in $\mathbb{R}_{>0}^{3}$ we have

$$
\begin{aligned}
\left\{\left(l_{i j}, l_{j k}, l_{k i}\right) \mid \cosh l_{i j}<\cosh l_{k i} \cosh l_{j k}+I_{i j} \sinh l_{k i} \sinh l_{j k}\right\} & \\
& \supset\left\{\left(l_{i j}, l_{j k}, l_{k i}\right) \mid l_{i j}<l_{k i}+l_{j k}\right\} .
\end{aligned}
$$

Applying the same argument in Euclidean geometry, we see $\rho_{H}\left(\mathbb{R}_{>0}^{3}\right) \cap \mathcal{L}$ is a cone. Therefore $\mathcal{R}_{H}^{i j k}=\rho_{H}^{-1}\left(\rho_{H}\left(\mathbb{R}_{>0}^{3}\right) \cap \mathcal{L}\right)$ is connected and simply connected. 
In the construction of the energy function, we will use a natural variable $u=$ $\left(u_{1}, u_{2}, \ldots, u_{|V|}\right)$ where $u_{i}=\ln \tanh \frac{r_{i}}{2}$. Let $\tau_{H}: \mathbb{R}^{3} \rightarrow \mathbb{R}_{>0}^{3}$ be the map sending $\left(u_{i}, u_{j}, u_{k}\right)$ to $\left(r_{i}, r_{j}, r_{k}\right)$. Since $\tau_{H}$ is a diffeomorphism, we have

Corollary 10. The set $\mathcal{U}_{H}^{i j k}:=\tau_{H}^{-1}\left(\mathcal{R}_{H}^{i j k}\right)$ is a connected and simply connected open subset in $\mathbb{R}_{>0}^{3}$.

To prove Theorem 2, we construct a strictly concave energy function on $\mathcal{U}_{H}^{i j k}$ for each triangle $\triangle i j k \in F$. As in the case of Euclidean geometry, the space $\mathcal{U}_{H}^{i j k}$ is not always convex (for example, when $I_{i j}=I_{j k}=I_{k i}=2$ ); we cannot obtain global rigidity. Therefore we are not able to determine the space of cone angles in the case of inversive distance circle packing.

3.2. Energy function. To prove Theorem 2, we will construct an energy function on the space of radius vectors. First, we construct an energy function on the space for one triangle. To simplify notation, consider the triangle $\triangle 123 \in F$ and the map

$$
\begin{array}{ccccccc}
\mathcal{U}_{H}^{123} & \rightarrow & \mathcal{R}_{H}^{123} & \rightarrow & \mathcal{L} & \rightarrow & \mathbb{R}_{>0}^{3}, \\
\left(u_{1}, u_{2}, u_{3}\right) & \mapsto & \left(r_{1}, r_{2}, r_{3}\right) & \mapsto & \left(l_{1}, l_{2}, l_{3}\right) & \mapsto & \left(\alpha_{1}, \alpha_{2}, \alpha_{3}\right),
\end{array}
$$

where $r_{i}=\ln \frac{1+e^{u_{i}}}{1-e^{u_{i}}}$ for $i=1,2,3$. For three fixed positive numbers $I_{12}, I_{23}, I_{31}$, we have $\cosh l_{i}=\cosh r_{j} \cosh r_{k}+I_{i j} \sinh r_{j} \sinh r_{k}$ for $\{i, j, k\}=\{1,2,3\}$. For simplicity of notation, we set $\left(l_{1}, l_{2}, l_{3}\right)=\left(l_{23}, l_{31}, l_{12}\right)$. Also, $\alpha_{1}, \alpha_{2}, \alpha_{3}$ are inner angles of a hyperbolic triangle with edge lengths $l_{1}, l_{2}, l_{3}$. By the cosine law,

$$
\cos \alpha_{i}=\frac{-\cosh l_{i}+\cosh l_{j} \cosh l_{k}}{\sinh l_{j} \sinh l_{k}}
$$

for $\{i, j, k\}=\{1,2,3\}$. Therefore $\alpha_{1}, \alpha_{2}, \alpha_{3}$ are functions in terms of $u_{1}, u_{2}, u_{3}$.

Lemma 11. The Jacobian matrix of functions $\alpha_{1}, \alpha_{2}, \alpha_{3}$ in terms of $u_{1}, u_{2}, u_{3}$ is symmetric.

Proof. Due to the cosine law of a hyperbolic triangles, $\alpha_{1}, \alpha_{2}, \alpha_{3}$ are functions of $l_{1}, l_{2}, l_{3}$. The following formula can be proved by direct calculation. For $\{i, j, k\}=$ $\{1,2,3\}$,

$$
\begin{aligned}
\left(\begin{array}{c}
d \alpha_{1} \\
d \alpha_{2} \\
d \alpha_{3}
\end{array}\right)=\frac{-1}{\sin \alpha_{i} \sinh l_{j} \sinh l_{k}}\left(\begin{array}{ccc}
\sinh l_{1} & 0 & 0 \\
0 & \sinh l_{2} & 0 \\
0 & 0 & \sinh l_{3}
\end{array}\right) \\
\times\left(\begin{array}{ccc}
-1 & \cos \alpha_{3} & \cos \alpha_{2} \\
\cos \alpha_{3} & -1 & \cos \alpha_{1} \\
\cos \alpha_{2} & \cos \alpha_{1} & -1
\end{array}\right)\left(\begin{array}{c}
d l_{1} \\
d l_{2} \\
d l_{3}
\end{array}\right) .
\end{aligned}
$$

Next, by the construction $\cosh l_{i}=\cosh r_{j} \cosh r_{k}+I_{j k} \sinh r_{j} \sinh r_{k}$, for $\{i, j, k\}$ $=\{1,2,3\}$, where $I_{i j}$ is a constant. Differentiating the two sides of the equality, we obtain

$$
d l_{i}=\frac{-\cosh r_{k}+\cosh l_{i} \cosh r_{j}}{\sinh l_{i} \sinh r_{j}} d r_{j}+\frac{-\cosh r_{j}+\cosh l_{i} \cosh r_{k}}{\sinh l_{i} \sinh r_{k}} d r_{k}
$$

Finally, $r_{i}=\ln \frac{1+e^{u_{i}}}{1-e^{u_{i}}}$ implies $\sinh r_{i} d u_{i}=d r_{i}, i=1,2,3$. 
Combining the three relations, we have, for $\{i, j, k\}=\{1,2,3\}$,

$$
\begin{aligned}
& \left(\begin{array}{l}
d \alpha_{1} \\
d \alpha_{2} \\
d \alpha_{3}
\end{array}\right)=\frac{-1}{\sin \alpha_{i} \sinh l_{j} \sinh l_{k}} \\
& \times\left(\begin{array}{ccc}
\sinh l_{1} & 0 & 0 \\
0 & \sinh l_{2} & 0 \\
0 & 0 & \sinh l_{3}
\end{array}\right)\left(\begin{array}{ccc}
-1 & \cos \alpha_{3} & \cos \alpha_{2} \\
\cos \alpha_{3} & -1 & \cos \alpha_{1} \\
\cos \alpha_{2} & \cos \alpha_{1} & -1
\end{array}\right) \\
& \times\left(\begin{array}{ccc}
\frac{-\cosh r_{3}+\cosh l_{1} \cosh r_{2}}{\sinh l_{1} \sinh r_{2}} & \frac{-\cosh r_{2}+\cosh l_{1} \cosh r_{3}}{\sinh l_{1} \sinh r_{3}} \\
\frac{-\cosh r_{3}+\cosh l_{2} \cosh r_{1}}{\sinh l_{2} \sinh r_{1}} & 0 & \frac{-\cosh r_{1}+\cosh l_{2} \cosh r_{3}}{\sinh l_{2} \sinh r_{3}} \\
-\cosh r_{2}+\cosh l_{3} \cosh r_{1} & \frac{-\cosh r_{1}+\cosh l_{3} \cosh r_{2}}{\sinh l_{3} \sinh r_{2}} & 0 \\
\hline \sinh l_{3} \sinh r_{1} & 0 \\
0 & \sinh r_{2} & 0 \\
0 & \sinh r_{3}
\end{array}\right)\left(\begin{array}{c}
d u_{1} \\
d u_{2} \\
d u_{3}
\end{array}\right) .
\end{aligned}
$$

We write the above formula as

$$
\left(\begin{array}{c}
d \alpha_{1} \\
d \alpha_{2} \\
d \alpha_{3}
\end{array}\right)=\frac{-1}{\sin \alpha_{i} \sinh l_{j} \sinh l_{k}} M\left(\begin{array}{c}
d u_{1} \\
d u_{2} \\
d u_{3}
\end{array}\right),
$$

where $M$ is a product of four matrixes.

To show that the Jacobian matrix of functions $\alpha_{1}, \alpha_{2}, \alpha_{3}$ in terms of $u_{1}, u_{2}, u_{3}$ is symmetric is equivalent to showing that the matrix $M$ is symmetric.

To simplify notation, let $a:=\cosh l_{1}, b:=\cosh l_{2}, c:=\cosh l_{3}, x:=\cosh r_{1}, y:=$ $\cosh r_{2}, z:=\cosh r_{3}$. By the cosine law, we have

$$
M=\left(\begin{array}{ccc}
1-a^{2} & a b-c & c a-b \\
a b-c & 1-b^{2} & b c-a \\
c a-b & b c-a & 1-c^{2}
\end{array}\right)\left(\begin{array}{ccc}
\frac{1}{a^{2}-1} & 0 & 0 \\
0 & \frac{1}{b^{2}-1} & 0 \\
0 & 0 & \frac{1}{c^{2}-1}
\end{array}\right)\left(\begin{array}{ccc}
0 & a y-z & a z-y \\
b x-z & 0 & b z-x \\
c x-y & c y-x & 0
\end{array}\right) .
$$

We check that $M$ is symmetric, for example, by showing

$$
M_{12}=M_{21}=z-\frac{a c-b}{c^{2}-1} x-\frac{b c-a}{c^{2}-1} y .
$$

Lemma 12. The Jacobian matrix of functions $\alpha_{1}, \alpha_{2}, \alpha_{3}$ in terms of $u_{1}, u_{2}, u_{3}$ is negative definite.

Proof. Using the notation of Lemma 11, we need to verify that the matrix $M$ is positive definite. The determinant of $M$ is positive since the determinant of each factor is positive. Note that the second matrix is a Gram matrix up to the negative sign. Since, by Corollary 10 , the set $\mathcal{U}_{H}^{123}$ is connected, to show that $M$ is positive definite, it is enough to check that $M$ is positive definite at one vector in $\mathcal{U}_{H}^{123}$.

It is equivalent to picking up a vector $\left(r_{1}, r_{2}, r_{3}\right)$ such that the resulting $l_{1}, l_{2}, l_{3}$ satisfy the triangle inequality. We pick up $\left(r_{1}, r_{2}, r_{3}\right)=(s, s, s)$. In the following, we show that such kind of number $s$ exists.

For fixed $I_{12}, I_{23}, I_{31} \in[0, \infty)$, we have $\cosh l_{i}=\cosh r_{j} \cosh r_{k}+I_{j k} \sinh r_{j} \sinh r_{k}=$ $\cosh ^{2} s+I_{j k} \sinh ^{2} s \geq \cosh ^{2} s$, for $\{i, j, k\}=\{1,2,3\}$. Therefore $\sinh l_{i} \geq \sqrt{\cosh ^{4} s-1}$ for $i=1,2,3$. 
The fact that $l_{1}, l_{2}, l_{3}$ satisfy the triangle inequality is equivalent to

$$
\begin{aligned}
& \cosh \left(l_{i}+l_{j}\right)>\cosh l_{k} \\
\Longleftrightarrow & \cosh l_{i} \cosh l_{j}+\sinh l_{i} \sinh l_{j}>\cosh l_{k}=\cosh ^{2} s+I_{i j} \sinh ^{2} s \\
\Longleftarrow & \cosh ^{4} s+\cosh ^{4} s-1>\cosh ^{2} s+I_{i j} \sinh ^{2} s .
\end{aligned}
$$

Let $x:=\cosh ^{2} s$. The above inequality is equivalent to $\left(2 x-I_{i j}+1\right)(x-1)>0$. Since $x>1$, we need $x>\frac{I_{i j}-1}{2}$.

Therefore $l_{1}, l_{2}, l_{3}$ as functions of $(s, s, s)$ satisfy the triangle inequality if $\cosh ^{2} s$ $>\max \left\{\frac{I_{12}-1}{2}, \frac{I_{23}-1}{2}, \frac{I_{31}-1}{2}\right\}$.

Now it is enough to check that $M$ is positive definite at such a vector $(s, s, s)$ when $s$ is sufficiently large. In this case,

$$
\begin{aligned}
M= & \left(\begin{array}{ccc}
\sinh l_{1} & 0 & 0 \\
0 & \sinh l_{2} & 0 \\
0 & 0 & \sinh l_{3}
\end{array}\right)\left(\begin{array}{ccc}
-1 & \cos \alpha_{3} & \cos \alpha_{2} \\
\cos \alpha_{3} & -1 & \cos \alpha_{1} \\
\cos \alpha_{2} & \cos \alpha_{1} & -1
\end{array}\right) \\
& \times\left(\begin{array}{ccc}
0 & \frac{-1+\cosh l_{1}}{\sinh l_{1}} & \frac{-1+\cosh l_{1}}{\sinh l_{1}} \\
\frac{-1+\cosh l_{2}}{\sinh l_{2}} & 0 & \frac{-1+\cosh l_{2}}{\sinh l_{2}} \\
\frac{-1+\cosh l_{3}}{\sinh l_{3}} & \frac{-1+\cosh l_{3}}{\sinh l_{3}} & 0
\end{array}\right) \cosh s:=M_{1} \cosh s .
\end{aligned}
$$

The fact that $M$ is positive definite is equivalent to $M_{1}$ being positive definite. Since $M_{1}$ only involves $l_{1}, l_{2}, l_{3}$, the fact that $M_{1}$ is positive definite is a property of a hyperbolic triangle. In fact, the fact that $M_{1}$ is positive definite was proved in the paper of Guo-Luo [13] (Lemma 4.4) by showing that its leading principal $1 \times 1$ and $2 \times 2$ minor is positive. For completeness, we include a proof here.

That the leading principal $1 \times 1$ minor is positive is equivalent to

$$
\cos \alpha_{3} \frac{-1+\cosh l_{2}}{\sinh l_{2}}+\cos \alpha_{2} \frac{-1+\cosh l_{3}}{\sinh l_{3}}>0
$$

Replacing $\alpha$ 's by l's by the cosine law, we obtain

$$
\cosh l_{1}>\frac{\cosh ^{2} l_{2}+\cosh ^{2} l_{3}+\cosh l_{2}+\cosh l_{3}}{2 \cosh l_{2} \cosh l_{3}+\cosh l_{2}+\cosh l_{3}} .
$$

Since $l_{1}>\left|l_{2}-l_{3}\right|$, therefore $\cosh l_{1}>\cosh \left(l_{2}-l_{3}\right)$. To show that (8) holds, it is enough to check that

(9) $\cosh l_{2} \cosh l_{3}-\sinh l_{2} \sinh l_{3} \geq \frac{\cosh ^{2} l_{2}+\cosh ^{2} l_{3}+\cosh l_{2}+\cosh l_{3}}{2 \cosh l_{2} \cosh l_{3}+\cosh l_{2}+\cosh l_{3}}$.

We simplify the notation by introducing $a:=\cosh l_{2}>1, b:=\cosh l_{3}>1$. Then (9) is rewritten as

$$
a b-\sqrt{\left(a^{2}-1\right)\left(b^{2}-1\right)} \geq \frac{a^{2}+b^{2}+a+b}{2 a b+a+b} .
$$

(10) is equivalent to

$$
a b-\frac{a^{2}+b^{2}+a+b}{2 a b+a+b} \geq \sqrt{\left(a^{2}-1\right)\left(b^{2}-1\right)} .
$$


Since $a>1, b>1$, the left hand side of (11) is positive. To show that (11) holds, we square the two sides and simplify. We have

$$
\begin{aligned}
& \left(a b^{4}+a^{4} b-a^{3} b^{2}-a^{2} b^{3}\right)+\left(a^{4}+b^{4}-2 a^{2} b^{2}\right)+\left(a^{3}+b^{3}-a b^{2}-a^{2} b\right) \geq 0 \\
\Longleftrightarrow & (a b+1)\left(a^{3}+b^{3}-a^{2} b-a b^{2}\right)+\left(a^{2}-b^{2}\right)^{2} \geq 0 \\
\Longleftrightarrow & (a b+1)(a+b)(a-b)^{2}+\left(a^{2}-b^{2}\right)^{2} \geq 0 .
\end{aligned}
$$

This shows that the leading principal $1 \times 1$ minor is positive.

To show that the leading principal $2 \times 2$ minor of $M_{1}$ is positive, for simplifying notation, let $t_{i}:=\frac{-1+\cosh l_{i}}{\sinh l_{i}}$. Up to a diagonal matrix, $M_{1}$ is

$$
\left(\begin{array}{ccc}
-1 & \cos \alpha_{3} & \cos \alpha_{2} \\
\cos \alpha_{3} & -1 & \cos \alpha_{1} \\
\cos \alpha_{2} & \cos \alpha_{1} & -1
\end{array}\right)\left(\begin{array}{ccc}
0 & t_{1} & t_{1} \\
t_{2} & 0 & t_{2} \\
t_{3} & t_{3} & 0
\end{array}\right) .
$$

That the leading principal $2 \times 2$ minor of $M_{1}$ is positive is equivalent to

$$
\begin{aligned}
& \left(t_{2} \cos \alpha_{3}+t_{3} \cos \alpha_{2}\right)\left(t_{1} \cos \alpha_{3}+t_{3} \cos \alpha_{1}\right)-\left(-t_{2}+t_{3} \cos \alpha_{1}\right)\left(-t_{1}+t_{3} \cos \alpha_{2}\right)>0 \\
& \Longleftrightarrow\left(\cos ^{2} \alpha_{3}-1\right) t_{1} t_{2}+\left(\cos \alpha_{1} \cos \alpha_{3}+\cos \alpha_{2}\right) t_{2} t_{3}+\left(\cos \alpha_{2} \cos \alpha_{3}+\cos \alpha_{1}\right) t_{1} t_{3}>0 \\
& \Longleftrightarrow-\sin ^{2} \alpha_{3} t_{1} t_{2}+\cosh l_{2} \sin \alpha_{1} \sin \alpha_{3} t_{2} t_{3}+\cosh l_{1} \sin \alpha_{2} \sin \alpha_{3} t_{1} t_{3}>0 \\
& \Longleftrightarrow \cosh l_{2} \sinh l_{1} t_{2} t_{3}+\cosh l_{1} \sinh l_{2} t_{1} t_{3}>\sinh l_{3} t_{1} t_{2} \\
& \Longleftrightarrow \frac{\cosh l_{2} \sinh l_{1}}{t_{1}}+\frac{\cosh l_{1} \sinh l_{2}}{t_{2}}>\frac{\sinh l_{3}}{t_{3}} \\
& \Longleftrightarrow \cosh l_{2}\left(1+\cosh l_{1}\right)+\cosh l_{1}\left(1+\cosh l_{2}\right)>1+\cosh l_{3} \\
& \Longleftrightarrow\left(\cosh l_{1}+\cosh l_{2}-1\right)+\left(2 \cosh l_{1} \cosh l_{2}-\cosh l_{3}\right)>0 .
\end{aligned}
$$

The cosine law and the sine law of a hyperbolic triangle are used in the above calculation.

The last inequality is true since $\cosh l_{1}+\cosh l_{2}>1$ and $2 \cosh l_{1} \cosh l_{2}>$ $\cosh l_{1} \cosh l_{2}+\sinh l_{1} \sinh l_{2}=\cosh \left(l_{1}+l_{2}\right)>\cosh l_{3}$.

For the special case of intersection angle circle packing, Lemma 12 was proved in Chow-Luo 9] (Lemma 3.1) based on Thurston's observation 23 that the angle $\alpha_{i}$ is a decreasing function of $r_{i}$ and an increasing function of $r_{j}$ for $j \neq i$. But this observation does not hold in the general case of inversive distance circle packing. For example, when $l_{1}=2, l_{2}=2, l_{3}=3, r_{1}=r_{2}=r_{3}=1$, the matrix $M$ is

$$
\left(\begin{array}{ccc}
6.08 & 0.49 & -2.94 \\
0.49 & 6.08 & -2.94 \\
-2.94 & -2.94 & 22.11
\end{array}\right)
$$

with eigenvalue $23.15,5.59,5.53$. This shows that $\frac{\partial \alpha_{1}}{\partial r_{2}}<0$, while $\frac{\partial \alpha_{1}}{\partial r_{3}}>0$ for some value of $l_{i}$ and $r_{i}$.

Since, by Corollary [10, the space $\mathcal{U}_{H}^{123}$ of vectors $\left(u_{1}, u_{2}, u_{3}\right)$ is connected and simply connected, Lemma 11 and Lemma 12 imply

Corollary 13. The differential 1 -form $\sum_{i=1}^{3} \alpha_{i} d u_{i}$ is closed. For any $c \in \mathcal{U}_{H}^{123}$, the integration $w\left(u_{1}, u_{2}, u_{3}\right)=\int_{c}^{\left(u_{1}, u_{2}, u_{3}\right)} \sum_{i=1}^{3} \alpha_{i} d u_{i}$ is a strictly concave function on $\mathcal{U}_{H}^{123}$ and satisfying, for $i=1,2,3$,

$$
\frac{\partial w}{\partial u_{i}}=\alpha_{i}
$$


Proof of Theorem 2 in hyperbolic geometry. Let's prove the local rigidity of inversive distance circle packing in hyperbolic geometry. For a weighted triangulated closed surface $(\Sigma, T, I)$ with $I_{i j} \in[0, \infty)$ for each $i j \in E$, let $\mathcal{U}_{H}$ be the open subset of $\mathbb{R}^{|V|}$ formed by the vectors $u=\left(u_{1}, u_{2}, \ldots, u_{|V|}\right)$ satisfying $\left(u_{i}, u_{j}, u_{k}\right) \in \mathcal{U}_{H}^{i j k}$ whenever $\triangle i j k \in F$.

By Corollary [13, for each triangle $\triangle i j k \in F$, there is a function $w\left(u_{i}, u_{j}, u_{k}\right)$. Define a function $W: \mathcal{U}_{H} \rightarrow \mathbb{R}$ by

$$
W\left(u_{1}, u_{2}, \ldots, u_{|V|}\right)=\sum_{\triangle i j k \in F} w\left(u_{i}, u_{j}, u_{k}\right),
$$

where the sum is over all triangles in $F$. By Corollary $13, W$ is strictly concave on $\mathcal{U}_{H}$ so that $\frac{\partial W}{\partial u_{i}}$ equals the sum of inner angles having vertex $i$, i.e., the cone angle at vertex $i$. That means the gradient of $W$ is exactly the map sending a vector $u$ to its cone angles.

The local rigidity of inversive distance circle packing in hyperbolic geometry holds due to Lemma 8

\section{ACKNOWLEDGMENT}

The author would like to thank Feng Luo and Albert Madern for encouragement and helpful comments. He also thanks the referee for valuable suggestions.

\section{REFERENCES}

1. E. M. Andreev, Convex polyhedra in Lobachevsky spaces. (Russian) Mat. Sb. (N.S.) 81 (123) 1970 445-478. MR 0259734(41:4367)

2. E. M. Andreev, Convex polyhedra of finite volume in Lobachevsky space. (Russian) Mat. Sb. (N.S.) 83 (125) 1970 256-260. MR.0273510(42:8388)

3. W. Brägger, Kreispackungen und Triangulierungen. Enseign. Math., 38:201-217, 1992. MR:1189006 (94b:52032)

4. P. L. Bowers, M. K. Hurdal, Planar conformal mappings of piecewise flat surfaces. Visualization and Mathematics III, 3-34, Math. Vis., Springer, Berlin, 2003. MR2046999

5. P. L. Bowers, K. Stephenson, Uniformizing dessins and Belyı maps via circle packing. Mem. Amer. Math. Soc. 170 (2004), no. 805. MR2053391(2005a:30068)

6. A. I. Bobenko, B. A. Springborn, Variational principles for circle patterns and Koebe's theorem. Trans. Amer. Math. Soc. 356 (2004), no. 2, 659-689. MR2022715 (2005b:52054)

7. H. S. M. Coxeter, Inversive distance. Ann. Mat. Pura Appl. (4) 711966 73-83. MR0203568 $(34: 3418)$

8. Y. Colin de Verdiére, Un principe variationnel pour les empilements de cercles. Invent. Math., 104:655-669, 1991. MR:1106755 (92h:57020)

9. B. Chow, F. Luo, Combinatorial Ricci flows on surfaces. J. Differential Geom. 63 (2003), no. 1, 97-129. MR2015261 (2005a:53106)

10. J. Dai, X. D. Gu, F. Luo, Variational principles for discrete surfaces. Advanced Lectures in Mathematics (ALM), 4. International Press, Somerville, MA; Higher Education Press, Beijing, 2008. MR 2439807

11. D. Glickenstein, Discrete conformal variations and scalar curvature on piecewise flat two and three dimensional manifolds. arXiv:0906.1560, to appear in J. Differential Geom.

12. R. Guo, A note on circle patterns on surfaces. Geom. Dedicata 125 (2007), 175-190. MR.2322547 (2008f:52027)

13. R. Guo, F. Luo, Rigidity of polyhedral surfaces, II. Geom. Topol. 13 (2009), 1265-1312. MR2496046

14. Z.-X. He, Rigidity of infinite disk patterns. Ann. of Math. (2) 149 (1999), no. 1, 1-33. MR 1680531 (2000j:30068)

15. M. K. Hurdal, K. Stephenson, Discrete conformal methods for cortical brain flattening. Neuroimage, vol. 45, S86-S98, 2009. 
16. P. Koebe, Kontaktprobleme der konformen Abbildung. Ber. Sächs. Akad. Wiss. Leipzig, Math.Phys. Kl. 88 (1936), 141-164.

17. G. Leibon, Characterizing the Delaunay decompositions of compact hyperbolic surface. Geom. Topol. 6 (2002), 361-391. MR.1914573 (2003c:52034)

18. F. Luo, Rigidity of polyhedral surfaces. arXiv:math.GT/0612714, to appear in J. Differential Geom.

19. A. Marden, B. Rodin, On Thurston's formulaton and proof of Andreeev's theorem. Computational methods and function theory (Valparaíso, 1989), 103-116, Lecture Notes in Math., 1435, Springer, Berlin, 1990. MR.1071766 (92b:52040)

20. I. Rivin, Euclidean structures of simplicial surfaces and hyperbolic volume. Ann. of Math., 139:553-580, 1994. MR:1283870 (96h:57010)

21. B. Springborn, A variational principle for weighted Delaunay triangulations and hyperideal polyhedra. J. Differential Geom. 78 (2008), no. 2, 333-367. MR.2394026 (2009a:52010)

22. K. Stephenson, Introduction to circle packing. The theory of discrete analytic functions. Cambridge University Press, Cambridge, 2005. MR2131318 (2006a:52022)

23. W. P. Thurston, Geometry and topology of 3-manifolds. lecture notes, Math. Dept. of Princeton University, 1979.

School of Mathematics, University of Minnesota, Minneapolis, Minnesota 55455

E-mail address: guoxx170@math.umn.edu 